\title{
Knowledge Discovery by Decision Tree to Model the Rate of Drug Release from Matrix Substrates
}

\author{
Parastoo Namdarian \\ Biomedical Engineering \\ Department, Science and \\ Research Branch, Islamic \\ Azad University, Tehran, Iran.
}

\author{
Azadeh Asefnejad \\ Biomedical Engineering \\ Department, Science and \\ Research Branch, Islamic \\ Azad University, Tehran, Iran.
}

\author{
Fardad Farokhi \\ Biomedical Engineering \\ Department, Central Tehran \\ Branch, Islamic Azad \\ University, Tehran, Iran.
}

\begin{abstract}
To study the effect of a drug on a target tissue, mainly all process of drug delivery from drug delivery systems to the transferred drug in whole body must be considered. These studies are very attractive for different fields of drug delivery systems and pharmaceutical industry. Modeling can help us for better understanding of this process and reaching to better design of system. In this paper, we extract the rules of drug delivery by decision tree for a specific systems such as Matrix substrate. The results show that we can achieve to \%98.04 and $\% 74.51$ accuracy, if we use decision tree as a classifier and a predictor respectively.
\end{abstract}

\section{Keywords}

Drug Release, Modeling, Decision Tree, Matrix Substrates

\section{INTRODUCTION}

The aim of release control systems is to achieve an optimal concentration of drug in the blood or target tissue for a time as long as possible. In the other words, control of release rate and duration of release must be considered as important factors. Increasing the effectiveness of drugs, patient comfort, decreasing the rate of administration and reduction of the side effects of wrong doses are the main reasons that cause this field of science as an interesting field.

This has led pharmacologists and engineers strive together to achieve an efficient product. In this context, modeling can help to predicate the release before the delivery system was made.

W. Mark Saltzman [1] introduce pharmacokinetic models in two parts: Compartmental Models and Physiological Models. Compartmental models can be considered as a simple one compartment or extent to multi compartments. In the case of one compartment, a first order differential equation can be used for drug mass. Also the drug absorption can be considered in one compartment. For more complex process, two compartment model must be used.

In physiological modeling of pharmacokinetic, model based on actual anatomic of components and physiological mechanisms associated with drug metabolism is founded. In this model, which contains many details, the final status of drug are evaluated by elimination rate and the rate of outflow from any of the body's organs.

Drug release kinetics modeling by controlling drug release systems are divided into two categories [5]:

\section{Statistical Methods \\ 2. Model Based Methods}

Exploratory data analysis statistical method can be used to compare the dissolution either graphically or numerically. Multivariate methods are based on iterative design criteria, that the solubility is the independent variable and the time is the dependent variable. Model-dependent methods are based on mathematical functions that describe the mechanism of dissolve. Some of these models are: Zero Order model, First Order model, Higuchi model, Hixson-Crowell model, Korsmeyer-Peppas model, Baker-Lonsdale model, Hopfenberg model, Gompertz model, Linear or first order regression model, Quadratic model or second order regression model and Nonlinear regression models

Adam M. persky et al [2] model the effect of ephedrine on the cardiovascular system. Their database consists of eight healthy volunteers, obese, non-smokers and aged 21 to 44 years. The data was fitted to a single component model in which the drug absorption was considered as a first order differential equation with time delay and also time drug elimination was considered as a first order differential equation. More than $95 \%$ accuracy in the concentration range 0.1 to $10 \mu g l^{-1}$ was reported.

Pharmacokinetics and pharmacodynamics for anesthesia drugs in children was studied by Rebecca Jacob et al. [3]

Calatayud et al. [4] have proposed on the protection mechanism for digestive system against oral nitroglycerin administration.

Dash et al. [5] have presented a mathematical model of drug release kinetics for drug delivery systems with the ability to have control over the dose.

Zhang et al. [6] have presented a mathematical model for Convection-enhanced. This method is used to treat brain tumors and measuring the effectiveness of this method is very important. Their model is based on conservation of mass and momentum.

Different drug delivery systems have been reviewed by Nicholas A. Peppas [8]. Karen $\mathrm{H}$ et al. [9] modeled the concentration distribution in brain tissue by Convectionenhanced delivery systems. Andrew D. et al. [10] developed a mathematical model of drug release using magnetic particles. The model includes flow of erythrocyte particles within microcirculations. The model is designed to follow the particles in a vascular network.

\section{MATERIALS AND METHODS}

The decision tree used in this study, in order to extract knowledge from the data base that collected from several drug delivery systems. From the extracted rules, a significant relationship between indicators of a field of science can be realized. Finding these rules can be useful in two areas. The first is that this knowledge can be used in the design of the drug delivery system in the pharmaceutical field. Second, the model based on this knowledge can help to have the ability of drug release prediction. 


\subsection{Database}

In this study, we need a database that contains the characteristics of drug delivery systems with a matrix substrate. The following features are very important in characteristics of drug delivery systems and can be used [12]:

1. Type of polymer: hydrogel, soluble, biodegradable, non-biodegradable, hydrophobic and hydrophilic

2. Porosity: large porous, small porous and nonporous.

3. Type of softeners

4. Initial drug concentration

5. Hardness

6. Friability

7. Molecular weight of polymer

8. Diffusion coefficient of polymer

9. Thickness

Different unit for same feature may be seen in database due to integration of different data set. For this reason the necessary transformation was performed to get the same unit for each of them. The approximated slope of drug release curve can be used as the output parameter.

The released amount of drug after 1 hour or after 8 hour was used in some articled as the output parameter. Several papers [12-27] in this area were examined. Delivery systems in these articles have been experimentally reported. Characteristics of these systems were studied and recorded.

These articles contain 94 different systems with different characteristics. The above features not are covered completely by these papers. Thus feature reduction is performed and the final features are: drug content, thickness, Friability, Hardness and type of polymer.

According to expert claim, there is not any redundancy in new feature domain. 52 systems from 94 systems have complete information about final features and output variables (Rate of release, Release after $1 \mathrm{hr}$. and Release after $8 \mathrm{hr}$.).

For discrete transformation on continues output variables, equal three bin was considered (\%0-\%33.33, \%33.33$\% 66.66, \% 66.66-\% 100)$.

\subsection{Decision Tree [11]}

The decision tree is a tree structure with each node (non-leaf node) represents a test on an attribute (or feature), every branch represents one of the possible results from performed test and each leaf node (or end node) represents the class label (or predicted value).

By using the decision tree, the following benefits can be obtained:

\section{Extraction "Rule Set" from decision tree}

2. Does not require any domain knowledge or parameter setting, thus it is appropriate for exploratory knowledge discovery

3. It can handle high dimensional data

4. It is intuitive

5. The learning and classification steps are simple and fast

\section{In general it have good accuracy}

Each pass from root node to any leaf represents a rule. A disjunction (logical OR) is implied between each of the extracted rules. Because the rules are extracted directly from the tree, they are mutually exclusive and exhaustive.

Another approach is using the predictor model. The output of a predictor model is numerical and for a classifier model is categorical. The decision tree structure in predictor model and classifier model is the same except that the leaf content of the predictor is a numerical value.

To prevent the overfitting, the decision tree must be pruned.

10-fold cross-validation used for evaluation of the results.

\section{RESULTS}

Because the statements in the antecedent and the consequent of a rule may be prolong, some symbolic letters are used for characteristics or values. Symbols used for features are summarized in Table 1.

Table (1): The Symbols used for features in rule statements

\begin{tabular}{|c|c|c|}
\hline Symbol & Feature (Unit) & Input/output \\
\hline $\mathrm{a}$ & Drug Content(\%) & Input \\
\hline $\mathrm{b}$ & Thickness(mm) & Input \\
\hline $\mathrm{c}$ & $\begin{array}{c}\text { Release Rate after } 8 \\
\text { hour(\%) }\end{array}$ & Output \\
\hline $\mathrm{d}$ & $\begin{array}{c}\text { Release Rate after 1 } \\
\text { hour(\%) }\end{array}$ & Output \\
\hline $\mathrm{e}$ & $\begin{array}{c}\text { Release Rate(\%/h) } \\
\text { friability }\end{array}$ & Output \\
\hline $\mathrm{f}$ & Input \\
\hline $\mathrm{g}$ & Hardness(kg/cm2) & Input \\
\hline $\mathrm{h}$ & Hydrogel Polymer & Input \\
\hline $\mathrm{j}$ & $\begin{array}{c}\text { Soluble Polymer } \\
\text { Polymer }\end{array}$ & Input \\
\hline $\mathrm{k}$ & $\begin{array}{c}\text { Non- Biodegradable } \\
\text { Polymer }\end{array}$ & Input \\
\hline $\mathrm{m}$ & $\begin{array}{c}\text { Hydrophobic } \\
\text { (Plastic) Polymer }\end{array}$ & Input \\
\hline $\mathrm{o}$ & $\begin{array}{c}\text { Hydrophilic } \\
\text { Polymer }\end{array}$ & Input \\
\hline
\end{tabular}

Five below rules are extracted for the slope of releasing curve with \%98.04 accuracy.

Only one rule for "High":

If $\mathrm{b}<=5.51 \& \mathrm{f}<=0.62 \& \mathrm{~m}=$ Hydrophobic Then e=High

Two rules for "Low":

If $m=$ Not Hydrophobic Then $\mathrm{e}=$ Low

If $b>5.52$ Then $e=$ Low

Two rules for " Medium ":

If $a<=98.57 \& b<=5.51 \& \mathrm{~m}=$ Hydrophobic Then $e=$ Medium

If $a>98.57 \& b>5.51 \& b<=5.52$ Then $e=$ Medium

Four below rules are extracted for releasing after $1 \mathrm{hr}$. with $\% 80$ accuracy. 
Only one rule for "High":

If $\mathrm{g}>6.2 \& \mathrm{~m}=$ Hydrophobic Then $\mathrm{d}=$ High

Two rules for "Low":

If $\mathrm{g}<=6.2 \& \mathrm{~m}=$ Hydrophobic Then $\mathrm{d}=$ Low

If $\mathrm{f}<=0.52$ Then $\mathrm{d}=$ Low

Only one rule for " Medium ":

If $\mathrm{f}>0.52 \& \mathrm{~m}=$ Not Hydrophobic Then $\mathrm{d}=$ Medium

Three below rules are extracted for releasing after $8 \mathrm{hr}$. with $\% 84.31$ accuracy.

Two rules for "High":

If $b>4.44$ Then $c=$ High

If $\mathrm{f}<=0.34$ Then $\mathrm{c}=$ High

Only one rule for " Medium ":

If $\mathrm{b}<=4.44$ Then $\mathrm{c}=$ Medium

For prediction model, 23 rules are extracted for the slope of releasing curve with $\% 74.51$ accuracy.

If $\mathrm{m}=$ Hydrophobic $\& \mathrm{f}>0.62$ Then $e=\% 2.18$

If $a>98.94 \& b<=3.13 \& f<=0.38$ Then $e=\% 4.76$

If $\mathrm{f}>0.42 \& \mathrm{f}<=0.57 \& \mathrm{i}=$ Not Soluble Polymer Then $\mathrm{e}=\% 5.21$

If $\mathrm{a}<=50$ Then $\mathrm{e}=\% 7.14$

If $b>5.19 \& \mathrm{f}>0.57 \& \mathrm{~g}>6.9 \& \mathrm{~m}=$ Not Hydrophobic Then $\mathrm{e}=\% 7.27$

If $b<=3.13 \& f>0.38 \& g>4.83$ Then $e=\% 8$

If $\mathrm{a}<=98.94 \& \mathrm{~b}<=3.13 \& \mathrm{f}<=0.42$ Then $\mathrm{e}=\% 8.33$

If $a>50 \& \mathrm{f}>0.42 \& \mathrm{i}=$ Soluble Polymer \& $\mathrm{m}=$ Not Hydrophobic Then $\mathrm{e}=\% 8.75$

If $b>4.44 \& \quad \mathrm{i}=$ Soluble Polymer $\& \mathrm{~m}=$ Not Hydrophobic Then $\mathrm{e}=\% 9.8$

If $\mathrm{f}<=0.42 \& \mathrm{~g}>7.035$ Then $\mathrm{e}=\% 10$

If $\mathrm{f}<=0.34 \& \mathrm{~g}<=5.17$ Then $\mathrm{e}=\% 11.25$

If $\mathrm{g}>5.17 \quad \& \quad \mathrm{~g}<=6.2 \& \mathrm{~h}=$ Not Hydrogel Polymer Then $\mathrm{e}=\% 12.5$

If $\mathrm{h}>0$ Then $\mathrm{e}=\% 13.33$

If $\mathrm{a}>98.8 \& \mathrm{~g}>5.17 \& \mathrm{~g}<=6.2$ Then $\mathrm{e}=\% 13.75$

If $b>3.13 \& f>0.34 \& g<=5.17$ Then $e=\% 14$

If $b>4.44 \& b<=5.2 \& i=$ Soluble Polymer Then $e=\% 14.7$

If $\mathrm{f}<=0.42 \& \mathrm{~g}>6.2 \& \mathrm{~g}<=7.035$ Then $\mathrm{e}=\% 17.5$

If $b<=5.19 \& f>0.42 \& i=$ Not Soluble Polymer Then $e=\% 21.7$

If $a>50 \& a<=98.94 \quad \& \quad b<=5.2 \& \quad f>0.42 \& i=$ Soluble Polymer $\& \mathrm{~m}=$ Not Hydrophobic Then $\mathrm{e}=\% 22$

If $\mathrm{f}>0.42$ Then $\mathrm{e}=\% 30$

If $\mathrm{b}<=5.51 \& \mathrm{f}<=0.62 \& \mathrm{~m}=$ Hydrophobic Then $\mathrm{e}=\% 44.4$

If $\mathrm{b}<=3.13 \& \mathrm{f}<=0.42 \& \mathrm{~g}<=4.83$ Then $\mathrm{e}=\% 46.66$

If $a>98.57 \& b<=5.51 \& f<=0.62 \& m=$ Hydrophobic Then $\mathrm{e}=\% 90$
For prediction model, 21 rules are extracted for the releasing after $1 \mathrm{hr}$. with $\% 60.78$ accuracy.

If $\mathrm{m}=$ Hydrophobic Then $\mathrm{d}=\% 5$

If $a<=98.94 \& \mathrm{f}<=0.38 \& \mathrm{~h}=$ Not Hydrogel Polymer Then $\mathrm{d}=\% 10$

If $a>98.94 \& \mathrm{f}<=0.38 \& \mathrm{i}=$ Not Soluble Polymer Then $\mathrm{d}=\% 15$

If $b<4.44 \& \mathrm{f}>0.42 \& \mathrm{~g}<=6.9 \& \mathrm{~m}=$ Not Hydrophobic Then $\mathrm{d}=\% 18$

If $\mathrm{h}=$ Hydrogel Polymer Then $\mathrm{d}=\% 23.15$

If $a>50 \& a<=98.05 \& \mathrm{f}>0.42 \& \mathrm{~m}=$ Not Hydrophobic Then $\mathrm{d}=\% 25$

If a $>99.8 \& \mathrm{f}<=0.42 \& \mathrm{i}=$ Soluble Polymer Then $\mathrm{d}=\% 25$

If $\mathrm{h}=$ Not Hydrogel Polymer $\& \mathrm{~m}=$ Not Hydrophobic Then $\mathrm{d}=\% 30$

If $a>98.67 \& f>0.42 \& g>6.9 \& m=$ Not Hydrophobic Then $\mathrm{d}=\% 35$

If $\mathrm{f}<=0.12$ Then $\mathrm{d}=\% 38$

If $\mathrm{f}>0.42 \& \mathrm{~g}<=6.9 \& \mathrm{i}=$ Not Soluble Polymer Then $\mathrm{d}=\% 40$

If $a<=99.8 \& \mathrm{f}>0.12 \& \mathrm{f}<=0.38 \& \mathrm{i}=$ Soluble Polymer Then $\mathrm{d}=\% 45$

If $\mathrm{f}>0.38 \& \mathrm{~g}<=4.67 \& \mathrm{~m}=$ Not Hydrophobic Then $\mathrm{d}=\% 45$

If $a>98.05 \& \mathrm{f}>0.42 \& \mathrm{~g}>6.9 \& \mathrm{i}=$ Soluble Polymer \& $\mathrm{m}=$ Not Hydrophobic Then $\mathrm{d}=\% 50$

If $b>4.44 \& \mathrm{~g}<=6.9 \& \mathrm{i}=$ Soluble Polymer Then $\mathrm{d}=\% 55$

If $\mathrm{f}<=0.62 \& \mathrm{~m}=$ Hydrophobic Then $\mathrm{d}=\% 60$

If $\mathrm{b}>5.52 \& \mathrm{~h}=$ Not Hydrogel Polymer Then $\mathrm{d}=\% 65$

If $\mathrm{b}<=5.51 \& \mathrm{f}<=0.62 \& \mathrm{~m}=$ Hydrophobic \& Then $\mathrm{d}=\% 70$

If $a>98.57 \& b>5.51 \& b<=5.52$ Then $d=\% 80$

If $a>98.57 \& b<=5.51 \& \mathrm{f}<=0.62 \& \mathrm{~m}=$ Hydrophobic Then $\mathrm{d}=\% 85$

For prediction model, 17 rules are extracted for the releasing after $8 \mathrm{hr}$. with $\% 60.78$ accuracy.

If $\mathrm{b}<=4.24 \& \mathrm{~m}=$ Hydrophobic Then $\mathrm{c}=\% 38$

If $\mathrm{a}>98.94 \& \mathrm{f}<=0.38 \& \mathrm{i}=$ Not Soluble Polymer Then $\mathrm{c}=\% 40$

If $\mathrm{a}<=98.94 \& \mathrm{~b}<=4.24 \& \mathrm{f}<=0.38$ Then $\mathrm{c}=\% 50$

If $a>99.43 \& b>3.03 \& b<=4.24 \& \quad f>0.38 \& g>4.83$ Then $\mathrm{c}=\% 60$

If $a>98.88 \&$ \& $<=99.43 \& \quad b<=4.24 \& f>0.38 \& m=$ Not Hydrophobic Then $\mathrm{c}=\% 65$

If $b<=3.03 \& f>0.38 \& m=$ Not Hydrophobic Then $c=\% 65$

If $\mathrm{f}<=0.38 \& \mathrm{i}=$ Soluble Polymer Then $\mathrm{c}=\% 70$

If $a<=98.05 \& b>4.24 \& i=$ Not Soluble Polymer Then $c=\% 78$

If $a>98.05 \& a<=98.88 \& b<4.24 \& f>0.38$ Then $c=\% 80.8$

If $\mathrm{f}<=0.12$ Then $\mathrm{c}=\% 83.92$

If $a<=99.8 \& \mathrm{f}>0.12 \& \mathrm{f}<=0.38 \& \mathrm{i}=$ Soluble Polymer Then $\mathrm{c}=\% 88.46$

If $b>4.24 \& b<=4.44$ Then $c=\% 90$ 
If $\mathrm{f}>0.38 \& \mathrm{~g}<=4.83 \& \mathrm{~m}=$ Not Hydrophobic Then $\mathrm{c}=\% 98.46$

If $b>4.44 \&$ i= Soluble Polymer \& $m=$ Not Hydrophobic Then $\mathrm{c}=\% 99$

If $\mathrm{b}>4.44$ Then $\mathrm{c}=\% 100$

\section{DISCUSSION}

From the rules that are extracted by decision tree, it is seen that the thickness and drug content from the proposed features are most important in the drug delivery which is in agreement with related studies in this area.

As was seen in the results, different accuracy obtained from $\% 60.78$ to \%98.04. It was expected that the accuracy in prediction mode be lower than the accuracy in classification mode, certainly due to the numerical format of the output in the prediction mode against the categorical (range) format of the output in the classification mode.

For release rate after eight hours, there is not any rules for "Low" and in the prediction mode there is not any rules with the output value under \%33. These two sentences are equivalent in meaning. In practice, drug delivery systems after this period of time release a large amount of the drug (or total amount of drug).

Unique approach in this research makes it very distinct from other methods. The main feature of this study is to provide a rule set for drug delivery systems. In other words, in addition to providing a behavioral model of drug delivery systems, Rules governing the release of the drug has been developed Which is useful and applicable for the different aspects, as mentioned in the introduction of article such as make of system, analysis of performance and etc.

\section{REFERENCES}

[1] W. Mark Saltzman, "Drug Delivery: Engineering Principles for Drug Therapy", Oxford University Press ,2001, page:175-196.

[2] Adam M. Persky, N. Seth Berry, Gary M. Pollack \& Kim L.R.Brouwer;" Modelling the Cardiovascular Effects of Ephedrine", British Journal of Clinical Pharmacology, 2004, 57:5, pp. 552-562.

[3] Rebecca Jacob, Krishnan B.S., Venkatesan, "Pharmacokinetics and Pharmacodynamics of anesthetic Drugs in Pediatrics", Indian Journal of Anesthesia, 2004, 48(5), pp. 340-346.

[4] Sara Calatayud, et al., "Mechanisms of gastroprotection by transdermal nitroglycerin in the rat", British Journal of Pharmacology, 1999, pp. 1111-1118.

[5] Suvakanta Dash, et al., "Kinetic Modeling on Drug Release from controlued Drug delivery Systems", Acta Poloniae Pharmaceutica-Drug Research, 2010, Vol. 67, No. 3, pp.217-223.

[6] Lujun Zhang, Mingqiang Yang, Mingyan Jiang a, "Mathematical Modeling for Convection-Enhanced Drug Delivery", 2012 International Workshop on Information and Electronics Engineering (IWIEE), Procedia Engineering 29 2012, pp. 268 - 274.

[7] Rongling Wu et al.," Mathematical modeling of systems pharmacogenomics towards personalized drug delivery", Advanced Drug Delivery Reviews, Elsevier, 65, 2013, pp. 903-904.
[8] Nicholas A. Peppas," Historical perspective on advanced drug delivery: How engineering design and mathematical modeling helped the field mature", Advanced Drug Delivery Reviews, Elsevier, 65, 2013, pp. 5-9.

[9] Karen H. Støverud, Melanie Darcis, Rainer Helmig, S. Majid Hassanizadeh, " Modeling Concentration Distribution and Deformation During ConvectionEnhanced Drug Delivery into Brain Tissue", Transp Porous Med, 2012, 92, pp.119-143.

[10] Andrew D. Grief, Giles Richardson, " Mathematical modelling of magnetically targeted drug delivery", Journal of Magnetism and Magnetic Materials, 293, 2005, pp.455-463.

[11] Jiawei Han \& Micheline Kamber, "Data Mining: Concepts and Techniques", Second Edition, 2006, ELSEVIER: MORGAN KAUFMANN PUBLISHERS.

[12] Pandya et al., "Matrix Type Drug Delivery System: A Review", International Journal for Pharmaceutical Research Scholars (IJPRS), Vol.1, No.4, 2012, 84-95.

[13] Md. Mofizur Rahman, et al.,"Effect of various grades of hydroxyl propyl methyl cellulose matrix systems as oral sustained release drug delivery systems for ranolazine", IJPI'sJournal of Pharmaceutics and Cosmetology, Vol 1: 2 (2011),81-92.

[14] Suresh V Kulkarni, et al., " Formulation and In Vitro Evaluation of Sustained Release Matrix Tablet of Zolpidem Tartrate", International Journal of PharmTech Research, Vol. 3, No.2, April-June 2011, pp 858-863.

[15] P. Mahajan, et al., " Valsartan release from sustained release matrix tablet and effect of cellulose derivatives", Int. J. of Pharm. \& Life Sci. (IJPLS), Vol. 2, Issue 1: Jan: 2011, 521-530.

[16] Mario Grassi, Gabriele Grassi," Mathematical Modelling and Controlled Drug Delivery: Matrix Systems", Current Drug Delivery, 2005, 2, 97-116.

[17] Silvina A. Bravo et al., " In-Vitro Studies of Diclofenac Sodium Controlled-release from Biopolymeric Hydrophilic Matrices", J Pharm Pharmaceut Sci, Vol.5, No.3, 2002, 213-219.

[18] Roohullah, et al.," Sustained Release Carbamezapine Matrix Tablets Prepared by Solvent-Evaporation Technique Using Different Polymers", Middle-East Journal of Scientific Research 15 (10): 1368-1374, 2013.

[19] Tavakoli et al. , " Evaluation of Nitroglycerin Sustain Release from a Microreservoir Transdermal Patch ", Asian J Pharm Clin Res, Vol 6 Suppl 5, 2013, 191-195.

[20] Somnath Sakore et al.," Formulation and evaluation of enalapril maleate sustained release matrix tablets", Int J Pharm Biomed Res 2013, 4(1), 21-26.

[21] Jacek Balcerzak et al.," Analysis of Model Drug Release Kinetics from Complex Matrices of PolylactideChitosan", Progress on Chemistry and Application of Chitin and Its Derivatives, Vol.15, 2010,117-126.

[22] Rajat Kar et al., " Formulation and In Vitro Characterization of Xanthan Gum-Based Sustained Release Matrix Tables of Isosorbide-5- Mononitrate", Iranian Journal of Pharmaceutical Research (2010), 9 (1): 13-19. 
[23] Lakshmana Prabu S et al ," Formulation and evaluation of oral sustained release of Diltiazem Hydrochloride using rosin as matrix forming material ", Ars Pharm, Vol. 50, No.,1, 2009; 32-42.

[24] Sandip B.Tiwari, " Modulation of Drug Release from Hydrophilic Matrices", Pharmaceutical Technology Europe, 2008.

[25] Marina Levina et al.," Application of a modelling system in the formulation of extended release hydrophilic matrices", Pharmaceutical Technology Europe, 2006.
[26] Uttam Mandal, et al., " Formulation and Optimization of sustained release matrix tablet of metformin HCI 500mg Using Response surface methodology", Yakugaku Zasshi, Vol. 127, No. 8, 2007, 1281-1290

[27] Xu Tongwen, He Binglin," Mechanism of sustained drug release in diffusion-controlled polymer matrixapplication of percolation theory", International Journal of Pharmaceutics ,170 (1998) 139-149 